\begin{tabular}{lllll}
\multicolumn{4}{l}{ Abstract } & PWE-61 Table 1 \\
\hline $\begin{array}{l}\text { Trial } \\
\text { format }\end{array}$ & $\begin{array}{l}\text { Pre/post } \\
\text { pandemic } \\
\text { (April 2020) }\end{array}$ & $\begin{array}{l}\text { Number } \\
\text { sites }\end{array}$ & $\begin{array}{l}\text { Recruitment rate } \\
\text { per month }\end{array}$ & $\begin{array}{l}\text { Recruitment rate per } \\
\text { month per site }\end{array}$ \\
\hline $\begin{array}{l}\text { SDT } \\
\text { (typical) }\end{array}$ & Pre-pandemic & 27 & 11.7 & 0.43 \\
$\begin{array}{l}\text { SDT } \\
\text { (enhanced) }\end{array}$ & Pre-pandemic & 1 & 2 & 2 \\
* & Post- & 26 & 3.6 & 0.14 \\
SDT & $\begin{array}{l}\text { pandemic } \\
\text { Post- } \\
\text { pandemic }\end{array}$ & 1 & 23.0 & 23.0 \\
VT & & & \\
\hline
\end{tabular}

*enhanced recruitment techniques including federation-level GP searches, consent-for-contact registries, and advanced media promotions.

recruitment efficacy and study compliance (drop-outs) in the two forms of the study.

Methods RELIEVE IBS-D is a commercially sponsored doubleblind, placebo-controlled, multi-centre study to test the efficacy and safety of intestinal adsorbents in diarrhoea predominant IBS (IBS-D). Recruitment began in November 2018 with a target to recruit 430 participants across 28 sites. In April 2020 , with approximately half the target reached, the pandemic paused or stopped recruitment in many sites. A single site (Newcastle) developed a virtual recruitment process that commenced in September 2020. In total 269 patients were randomised via the SDT and 161 via the VT. Recruitment rates were compared for the SDT pre-pandemic, open sites continuing to recruit SDT post-pandemic and a single site (Newcastle) running a VT. Drop-out rates of the first 80 cases completing the RCT phase of the SDT and VT were compared.

Results In April 2020, 232 participants (53.9\%) had been randomised before the study was paused.

VT started in September 2020 and target achieved in April 2021. Recruitment rates for different periods are in the table.

Analysis of study compliance ( 8 weeks) of the first 80 sequentially randomised participants showed a drop-out rate of $13 / 80(16 \%)$ for the SDT and 4/80 (5\%) for the VT $(\mathrm{p}<0.05)$.

Conclusions Enhanced recruitment techniques can significantly increase recruitment rates. However, the geographical exclusion created by SDTs limits the effectiveness of recruitment, which can be enhanced many-fold by a VT design. Preliminary evidence suggests that VTs may provide lower levels of patient withdrawals. The success of this study has been facilitated by close cooperation of the sponsor and NHS delivery site, together with involvement of patients in trial design.

\section{PWE-62 REPORTING OF COMORBIDITIES AND OVERLAP SYNDROMES IN FUNCTIONAL DYSPEPSIA: REVIEW OF 15 YEARS OF RCTS}

${ }^{1}$ Fabien Wuestenberghs*, ${ }^{2}$ Maria P Eugenicos. 'Université catholique de Louvain; CHU UCL Namur, Department of Gastroenterology and Hepatology, Yvoir, Belgium; ${ }^{2}$ University of Edinburgh; Western General Hospital, Department of Gastroenterology, Edinburgh, UK
Introduction The Rome Foundation stated recommendations in 2016 to report confounding factors in clinical trials concerning functional gastrointestinal disorders (FGIDs) [1]. The aim of our study was to assess the reporting of different comorbidities and overlap syndromes in patients with functional dyspepsia (FD).

Methods Randomised controlled trials (RCTs) that included adult patients with FD according to Rome criteria were selected via MEDLINE (PubMed ${ }^{\circledR}$ ) and Scopus between 2006 and 2021. Characteristics of the trials and the patients, exclusion criteria, psychological factors, other FGIDs, somatic disorders, and non-gastrointestinal (GI) diseases were all recorded. We analysed the level of reporting of these comorbidities, focussing on the most frequent (gastro-oesophageal reflux disease (GORD), irritable bowel syndrome (IBS), anxiety, depression, Helicobacter pylori infection, diabetes mellitus). The study selection and data extraction were performed by FW; the systematic review was registered in PROSPERO (CRD42021235219).

Results 108 articles were included in the final analysis. Clinical trials were mainly conducted in Asia (76.9\%) and involved a complementary and alternative medicine treatment in $45.4 \%$ of the cases. The mean number of reported comorbidities was 2.44 ( $\pm 1.23 \mathrm{SD})$. Only one paper reported all six comorbidities. $96.3 \%$ of the papers reported at least one comorbidity. $22.2 \%$ of papers reported anxiety, and $24.1 \%$ reported depression. $63.0 \%$ of the papers reported an overlap with GORD and $43.5 \%$ with IBS. $H$. pylori gastritis and diabetes mellitus were reported in $54.6 \%$ and $37.0 \%$ of the papers respectively. There was no reported overlap with non-GI comorbidities, but for headaches that were reported in $2.8 \%$ of the papers. Representation of the reporting of different comorbidities is shown in Figure 1. The reporting of overlaps and comorbidities during the study period remained unchanged.

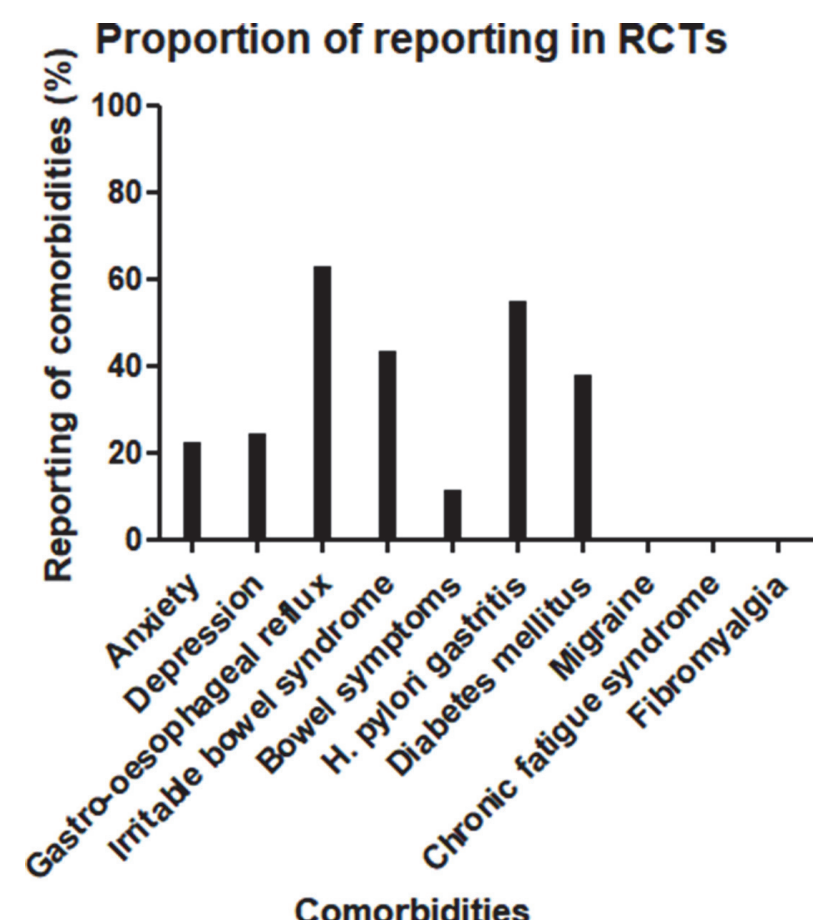

Abstract PWE-62 Figure 1 Representation of the proportion of the articles reporting each comorbidity 
Conclusions The most reported comorbidities in FD trials are depression and anxiety levels, overlaps with gastro-oesophageal reflux and IBS, H. pylori gastritis and diabetes mellitus. Overall, at least one of these co-morbidities was reported in almost all articles, with a mean of about 2.5 comorbidities reported in each one.

\section{REFERENCE}

1. Irvine EJ, Tack J, Crowell MD, Gwee KA, Ke M, Schmulson MJ, et al. Design of Treatment Trials for Functional Gastrointestinal Disorders. Gastroenterology 2016;150:1469-80 e1.

\section{PWE-63 RECRUITMENT POTENTIAL OF ENHANCED IBS TRIAL RECRUITMENT STRATEGIES IN THE COMMUNITY}

${ }^{1,2} \mathrm{Cho}$ Ee $\mathrm{Ng}^{*},{ }^{2} \mathrm{C}$ Howell, ${ }^{3} \mathrm{E}$ Markaryan, ${ }^{3} \mathrm{~A}$ Kemppinen, ${ }^{4} \mathrm{~L}$ Ling, ${ }^{2} \mathrm{~J}$ Newton, ${ }^{5} \mathrm{~J}$ McLaughlin, ${ }^{6} \mathrm{C}$ Knowles, ${ }^{1,7}$ Yan Yiannakou. ${ }^{1}$ University Hospital North Durham, Durham, UK; ${ }^{2}$ Newcastle University, Newcastle upon Tyne, UK; ${ }^{3}$ Enteromed Ltd, London, UK; ${ }^{4}$ University of Sunderland, Sunderland, UK; ${ }^{5}$ University of Manchester, Manchester, UK; ${ }^{6}$ Queen Mary University of London, London, UK; ${ }^{7}$ NIHR patient recruitment centre, Newcastle upon Tyne, UK

\subsection{6/gutjpl-2021-BSG.325}

Introduction Participant recruitment is a major factor to the success of a clinical trial and new strategies developed with the aim to increase community recruitment need to be evaluated. This is especially important in chronic conditions where patients are not routinely managed in specialist clinics. This study aims to describe the demographic of screened eligible individuals using the virtual trial process to an interventional irritable bowel syndrome (IBS) trial.

Methods The RELIEVE IBS-D trial was a commercially sponsored pragmatic trial assessing the efficacy of an over-thecounter intestinal adsorbent in the management of diarrhoea predominant irritable bowel syndrome (IBS-D). Following conversion to a virtual trial, consent for contact registries (C4CR), digital media (DM) and newspaper advertising (NA) were used to recruit participants. Potential participants were directed to an online web-based pre-screening questionnaire to assess their eligibility, before a virtual consultation and electronically consented.

Results $540(41.7 \%)$ of the 1292 screened population were eligible. C4CR identified participants had a different geographical distribution to those recruited through NA and DM, seen in figure 1.

Demographics were: C4CR mean age of $43 \pm 14.08$, with a male to female ratio of $1: 3$. DM, mean age $43.9 \pm 14.96$ with a male to female ratio of 2:7. NA, mean age was 60.6 \pm 16.59 and male to female ratio of $1: 2$.
Conclusions It is increasingly recognised that methods of reaching out to community-based participants is needed to improve recruitment to trials of some chronic conditions like IBS. Different recruitment modalities appear to appeal to different participant demographic. An effective 'recruitment engine' requires a multi-modality approach.

\section{PWE-64 UNDERSTANDING FACTORS THAT AFFECT RECRUITMENT TO AN INTERVENTIONAL TRIAL OF IRRITABLE BOWEL SYNDROME}

${ }^{1,2} \mathrm{Cho}$ Ee Ng, ${ }^{3} \mathrm{C}$ Howell, ${ }^{3}$ E Markaryan, ${ }^{3} \mathrm{~A}$ Kemppinen, ${ }^{4} \mathrm{~J} \mathrm{Ling},{ }^{2} \mathrm{~J}$ Newton, ${ }^{5} \mathrm{~J}$ McLaughlin, ${ }^{6} \mathrm{C}$ Knowles, ${ }^{1,7}$ Yan Yiannakou. ${ }^{1}$ University Hospital North Durham, Durham, UK; ${ }^{2}$ Newcastle University, Newcastle upon Tyne, UK; ${ }^{3}$ Enteromed Ltd, London, UK; ${ }^{4}$ University of Sunderland, Sunderland, UK; ${ }^{5}$ University of Manchester, Manchester, UK; ${ }^{6}$ Queen Mary University of London, London, UK; ${ }^{7}$ NIHR patient recruitment centre, Newcastle upon Tyne, UK

\subsection{6/gutjnl-2021-BSG.326}

Introduction Although functional bowel disorders are common, interventional therapy trials have often struggled to recruit to time and target. The challenges to trial recruitment include the fact that a high proportion of patients are not under specialist clinics; standard therapies are limited so that placebo control arms are unappealing; the ROME IV criteria are relatively restrictive. Understanding the factors that preclude eligibility is an important part of improving efficiency of trial recruitment.

Method The RELIEVE IBS-D trial was a commercially sponsored pragmatic trial assessing the efficacy of an over-thecounter intestinal adsorbent in the management of diarrhoea predominant irritable bowel syndrome (IBS-D). The second half of the trial, carried out during the pandemic, included a completely virtual trial management process; 233 patients were consented to the trial in this way. 1292 patients, who believed themselves to have IBS, volunteered for the virtual trial and were asked to undergo online eligibility screening followed by video-consultations. The reasons for failed eligibility were recorded in detail.

Results 233 (18\%) participants were consented out of 1292 that completed online screening. Table 1 describes the breakdown of criteria that limits eligibility.

Conclusions It is important that trial study cohorts represent the populations that will use the treatment. Virtual trial recruitment lends itself to detailed analysis of eligibility success due to the semi-automated algorithmic process employed. This study has shown that only 1 in 5 of those who believe they have IBS-D are eligible. The biggest cause of loss is the presence of significant levels of constipation symptom.
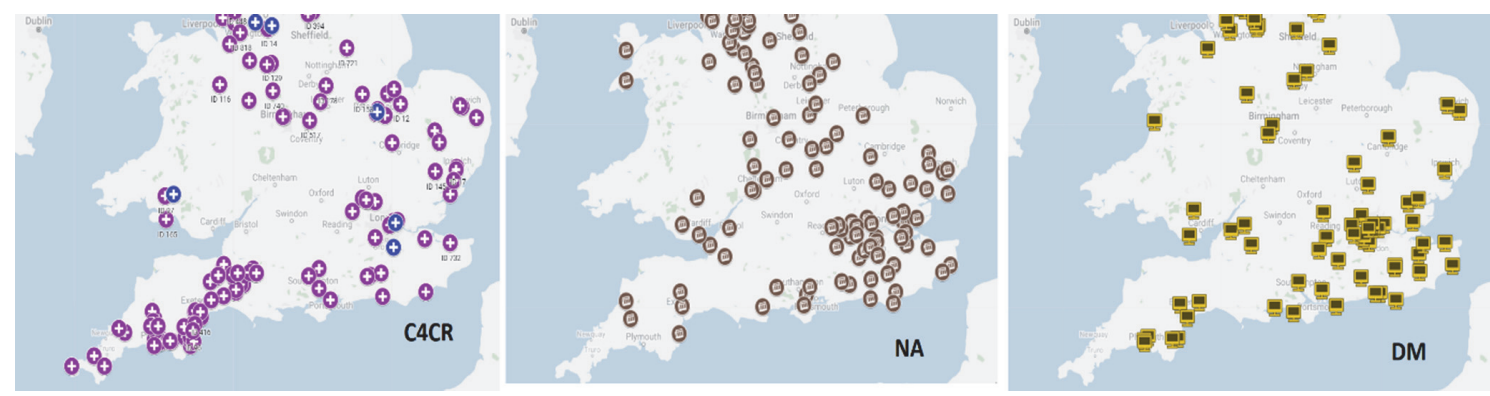